\title{
EIGENVALUE THEOREMS IN TOPOLOGICAL TRANSFORMATION GROUPS
}

\author{
BY \\ HARVEY B. KEYNES( $\left.{ }^{1}\right)$ AND JAMES B. ROBERTSON
}

1. Introduction. In this paper, we continue the type of study of ergodicity and weakly mixing that was initiated in [10]. Our framework is a general transformation group $(X, T, \pi)$, where the joint continuity of $(x, t) \rightarrow x \pi^{t}$ is not assumed. In $\S 2$, it is shown that most of the results of $[10, \S 3]$ are valid.in this setting when $X$ is simply a Baire space and eigenvalues are group characters. The conjecture in [10] concerning the weakly mixing theorem is partially answered by generalizing a result in Furstenberg [6, pp. 505-507]. In particular, it is shown that the existence of a certain type of ergodic measure is sufficient (Theorem 2.5). It is observed that both the minimal and uniquely ergodic transformation groups with a compact metrizable phase space satisfy this condition.

Applications of the results in $\S 2$ to various questions in topological dynamics on transformation groups with a continuous action are given in $\S 3$. It is shown that a minimal transformation group with compact metrizable phase space and abelian group has a trivial structure group precisely when it is weakly mixing. It follows that a minimal transformation group with any reasonable structure on the proximal relation has a nontrivial structure group. In addition, it is proved that if the above type of transformation group has a simply-connected, connected, locally pathconnected phase space, it must be weakly mixing. This implies that any minimal discrete or continuous flow on a sphere must be weakly mixing. Hence such flows are in a sense the extreme negation of the distal and equicontinuous ones.

Finally, $\S 4$ is concerned with eigenvalue theorems for minimal transformation groups. It is shown that the results corresponding to [10, $\$ 3]$ hold when the notion of equality almost everywhere is replaced by spatial equality.

The authors would like to thank the referee for a variety of helpful suggestions concerning this paper (e.g., Remark 3.6, (4)).

2. Eigenvalues. In this section we continue the study of eigenfunctions initiated in $[10, \S 3]$ and generalize the results given there. Throughout this section, $X$ will denote a Baire space (i.e., a topological space in which every comeager set is dense), $T$ an abstract group, with or without a topology, and $\pi: t \rightarrow \pi^{t}$ a homomorphism from $T$ into the group of all homeomorphisms of $X$ onto $X$ with composition as the group operation. We call $(X, T, \pi)$ a transformation group. If $T$ is a

Received by the editors April 5, 1968 and, in revised form, June 3, 1968.

( $\left.{ }^{1}\right)$ The work of this author was supported in part by NSF Grant GP-8394. 
topological group and if $(x, t) \rightarrow x \pi^{t}$ is continuous, we then say that $(X, T, \pi)$ is a continuous transformation group. We recall that $(X, T, \pi)$ is called ergodic if every proper closed invariant set is nowhere dense (i.e., if $C$ is a proper closed subset of $X$ such that $(C) \pi^{t} \subseteq C$ for all $t \in T$, then $C$ has empty interior), and that $(X, T, \pi)$ is called weakly mixing if $(X \times X, T, \pi \times \pi)$, defined by $(x, y)(\pi \times \pi)^{t}=\left(x \pi^{t}, y \pi^{t}\right)$, is ergodic.

As in $[10, \S 3] \mathscr{B}(X)$ will denote the algebra of all bounded complex-valued functions $f$ on $X$ such that $C(f)=\{x: f$ is continuous at $x\}$ is comeager. Two functions $f$ and $g$ in $\mathscr{B}(X)$ are said to be equal almost everywhere $(f=g$ a.e.) if $\{x: f(x)=g(x)\}$ is comeager. A transformation group $(X, T, \pi)$ induces a group of linear homomorphisms $\left\{L_{t}: t \in T\right\}$ on $\mathscr{B}(X)$ defined by $L_{t} f(x)=f\left(x \pi^{t}\right)$. We note that $f=g$ a.e. implies $L_{t} f=L_{t} g$ a.e. for all $t \in T$.

There are two notions of eigenfunctions in $\mathscr{B}(X)$. We say that $f \in \mathscr{B}(X)$ is an eigenfunction of $\left\{L_{t}: t \in T\right\}$ with eigenvalue $\chi$ if $f$ is not equal to 0 a.e. and if $L_{t} f$ $=\chi(t) f$ a.e. for all $t \in T$, where $\chi$ is a character of $T$. If $\chi$ is the trivial character $(\chi \equiv 1)$, then $f$ is said to be an invariant function. If $L_{t} f=\chi(t) f$ for all $t \in T$, then $f$ is called a spatial eigenfunction (or a spatially invariant function if $\chi$ is trivial). It is easily verified that a nonzero a.e. function $f$ in $\mathscr{B}(X)$ is a spatial eigenfunction (respectively eigenfunction) if and only if the linear subspace of $\mathscr{B}(X)$ (respectively $\mathscr{B}(X)$ modulo the ideal of functions equal to 0 a.e.) spanned by $f$ is invariant under $\left\{L_{t}: t \in T\right\}$. Also, if $(X, T, \pi)$ is a continuous transformation group, then the eigenvalues of spatial eigenfunctions are necessarily continuous and the eigenvalues of eigenfunctions are sequentially continuous. We do not know if the two concepts of eigenfunctions are actually distinct. However, we do have

2.1. Lemma. Let $f \in \mathscr{B}(X)$ be an invariant function. Then there exists a spatially invariant function $g \in \mathscr{B}(X)$ such that $f=g$ a.e.

Proof. Define $g$ by

$$
g(x)=\lim _{U \in \mathcal{N}_{x}}[\sup \{\operatorname{Re} f(y): y \in U \cap C(f)\}+i \sup \{\operatorname{Im} f(y): y \in U \cap C(f)\}]
$$

where $\mathscr{N}_{x}$ is the directed system of neighborhoods of $x$. The above limit exists since the two nets involved are bounded and monotonic. It is easy to see that $g(x)=f(x)$ for all $x \in C(f)$ and that $C(f) \subseteq C(g)$. Thus $g \in \mathscr{B}(X)$ and $f=g$ a.e. To see that $g$ is spatially invariant we note that

$$
\sup \{h(y): y \in U \cap C\}=\sup \{h(y): y \in U \cap C(h)\},
$$

where $h$ is any real-valued function in $\mathscr{B}(X)$ and $C$ is any dense subset of $C(h)$. Hence, taking

$$
C=C(f) \cap\left[\left\{x: L_{t} f(x)=f(x)\right\} \cap C(f)\right] \pi^{t},
$$


we obtain

$$
\begin{aligned}
L_{t} g(x) & =\lim _{U \in \mathscr{N}_{x \pi} t}[\sup \{\operatorname{Re} f(y): y \in U \cap C\}+i \sup \{\operatorname{Im} f(y): y \in U \cap C\}] \\
& =\lim _{U \in \mathscr{N}_{x \pi} t}\left[\sup \left\{\operatorname{Re} f\left(y \pi^{t}\right): y \pi^{t} \in U \cap C\right\}+i \sup \left\{\operatorname{Im} f\left(y \pi^{t}\right): y \pi^{t} \in U \cap C\right\}\right] \\
& =\lim _{V \in \mathscr{N}_{x}}\left[\sup \left\{\operatorname{Re} f(y): y \in V \cap C \pi^{-t}\right\}+i \sup \left\{\operatorname{Im} f(y): y \in V \cap C \pi^{-t}\right\}\right] \\
& =g(x) .
\end{aligned}
$$

The next two theorems generalize Theorems 3.1 and 3.7 respectively of [10].

2.2. TheOREM. A transformation group $(X, T, \pi)$ is ergodic if and only if every spatially invariant function in $\mathscr{B}(X)$ is equal to a constant almost everywhere.

Proof. Suppose $f \in \mathscr{B}(X)$ is spatially invariant. If $f$ is not equal to a constant a.e., then there exist two points $x$ and $y$ in $C(f)$ such that $f(x) \neq f(y)$. Then if $U$ is a closed neighborhood of $f(x)$ disjoint from $f(y), f^{-1}(U)^{-}$is a proper closed invariant set with nonempty interior. Hence $(X, T, \pi)$ is not ergodic.

If, on the other hand, $(X, T, \pi)$ is not ergodic, then the indicator function of a proper closed invariant set with nonempty interior is an invariant function in $\mathscr{B}(X)$ which is not equal to a constant almost everywhere. Q.E.D.

It follows from Lemma 2.1 and Theorem 2.2 that $(X, T, \pi)$ is ergodic if and only if every invariant function is equal to a constant almost everywhere. As a corollary, it follows that the proof of Theorem 3.5 of [10] concerning eigenvalues which are roots of unity generalizes to any Baire space.

2.3. THEOREM. If $(X, T, \pi)$ is weakly mixing, then every eigenfunction is equal to a constant almost everywhere.

Proof. Suppose $L_{t} f=\chi(t) f$ a.e. Consider $g \in \mathscr{B}(X \times X)$ defined by $g(x, y)$ $=f(x) f(y)^{*}$, where ${ }^{*}$ denotes the complex conjugate. Then $g$ is $\left\{L_{t} \times L_{t}: t \in T\right\}$ invariant and hence is equal to a constant a.e. However, if $f$ is not a constant a.e. there exist $x$ and $y$ in $C(f)$ such that $f(x) \neq 0$ and $f(x) \neq f(y)$. Then $(x, y)$ and $(x, x)$ are in $C(g)$, and $g(x, y)=f(x) f(y)^{*} \neq f(x) f(x)^{*}=g(x, x)$ which is a contradiction. Q.E.D.

For the next theorem we need

2.4. Lemma. Let $X$ be second countable and let $\mu$ be an invariant probability measure on the Borel sets of $X$ whose support is $X$. Then the following are equivalent:

(a) $\mu$ is closed ergodic, i.e., every closed invariant set has $\mu$ measure zero or one.

(b) Every set of positive measure contains a point with dense orbit.

(c) Every measurable spatial eigenfunction in $\mathscr{B}(X)$ which is not equal to a constant almost everywhere with respect to $\mu$-measure is not equal to a constant almost everywhere, and $(X, T, \pi)$ is ergodic.

Proof. (a) implies (b). Let $U_{1}, U_{2}, \ldots$ be a topology base for $X$. Then the set of points whose orbit is not dense is $\bigcup_{i=1}^{\infty} \mathcal{O}\left(U_{i}\right)^{\prime}$ where $\mathcal{O}(U)=\bigcup_{t \in T}\left(U \pi^{t}\right)$ is the orbit 
of $U$. But $\mathcal{O}\left(U_{i}\right)^{\prime}$ is a proper closed invariant set and has measure zero, since the complement has positive measure. Therefore the set of points whose orbit is not dense has measure zero.

(b) implies (c). By a theorem due to Kakutani (cf. [6, p. 506]) the set of points with dense orbit is contained in $C(f)$ where $f \in \mathscr{B}(X)$ is a spatial eigenfunction. Therefore if $f=c$ a.e., then the set of points where $f \neq c$ is contained in the set points whose orbit is not dense, and hence $f=c$ a.e. $(\mu)$. This proves the first part. Since $X$ contains a point with dense orbit, $(X, T, \pi)$ is ergodic.

(c) implies (a). Suppose $C$ is a proper closed invariant set. Since $(X, T, \pi)$ is ergodic, $C$ is nowhere dense. Hence $1_{C}=0$ a.e. Therefore, $1_{C}$ is a constant a.e. $(\mu)$. Since $\mu\left(C^{\prime}\right)>0, \mu(C)=0$. Q.E.D.

The following proof of a partial converse to Theorem 2.3 is an extension of a proof of Furstenberg (cf. [6, pp. 505-507]) and is the main result of this section. We differ from Furstenberg mainly in obtaining certain kernels for a larger class of transformation groups.

2.5. THEOREM. Suppose:

(a) $X$ is a separable metrizable Baire space.

(b) $T$ is abelian.

(c) There exists a closed ergodic invariant probability measure $\mu$ on the Borel sets of $X$ whose support is $X$.

Then if $(X, T, \pi)$ is not weakly mixing, there exists a spatial eigenfunction of $\left\{L_{t}: t \in T\right\}$ which is not equal to a constant almost everywhere.

Proof. Since $(X, T, \pi)$ is not weakly mixing, there exists a proper closed invariant subset $C$ of $X \times X$ which has nonempty interior. Then $D=\{(x, y):(y, x) \in C\}$ has the same properties. If $(C \cap D)^{0} \neq \varnothing$, let $f=1_{C \cap D}$ be the indicator function of $C \cap D$. If $(C \cap D)^{0}=\varnothing$, let $f=i\left(1_{C}-1_{D}\right)$. In either case it follows that:

(1) $f$ is $L_{\infty}(X, \mu) \cap \mathscr{B}(X)$;

(2) $f$ is a linear combination of upper semicontinuous functions;

(3) $f$ is conjugate symmetric, i.e., $f(y, x)=f(x, y)^{*}$;

(4) $f$ is not equal to a constant a.e.;

(5) $f$ is spatially invariant under $\left\{L_{t} \times L_{t}: t \in T\right\}$.

It follows from (1) and (3) that $F$, defined by

$$
F(g)(x)=\int_{X} f(x, y) g(y) d \mu(y)
$$

is a Hilbert-Schmidt operator on $L_{2}(X, \mu)$ (cf. [ $\beta$, p. 242]). By (1) and (4) and the fact that the support of $\mu$ is $X$, it can be seen that $f$ is not equal to a constant a.e. $(\mu)$. Hence the range of $F$ contains functions that are not equal to a constant a.e. $(\mu)$. From (1) and (2) and the hypothesis on $X$, it follows that functions in the range of $F$ are bounded and are linear combinations of upper semicontinuous functions and hence are in $\mathscr{B}(X)$. 
Let $\lambda \neq 0$ be an eigenvalue of $F$ corresponding to some nonconstant eigenfunction. Let $H_{\lambda}$ be the set of functions of the form $\lambda^{-1} F(g)$ where $g$ is an eigenfunction of $F$ with eigenvalue $\lambda$. The elements of $H_{\lambda}$ are spatial eigenfunctions of $F$ with eigenvalue $\lambda$ and are in one-to-one correspondence with the elements of $L_{2}(X, \mu)$ (equivalence classes of functions) which are eigenfunctions of $F$ with eigenvalue $\lambda$. Since $F$ is a Hilbert-Schmidt operator, it then follows that $H_{\lambda}$ is a finite-dimensional subspace of $\mathscr{B}(X)$. The following equalities show that $F$ and $\left\{L_{t}: t \in T\right\}$ commute spatially:

$$
\begin{aligned}
L_{t} F g(x) & =\int_{X} f\left(x \pi^{t}, y\right) g(y) d \mu(y) \\
& =\int_{X} f\left(x \pi^{t}, y \pi^{t}\right) g\left(y \pi^{t}\right) d \mu(y) \\
& =\int_{X} f(x, y) g\left(y \pi^{t}\right) d \mu(y) \\
& =F L_{t} g(x) .
\end{aligned}
$$

Hence $\left\{L_{t}: t \in T\right\}$ maps $H_{\lambda}$ into $H_{\lambda}$. Also $\left\{L_{t}: t \in T\right\}$ is a group of unitary operators since

$$
\begin{aligned}
\left\|L_{t} g\right\|^{2} & =\int_{X} g\left(x \pi^{t}\right) g\left(x \pi^{t}\right)^{*} d \mu(x) \\
& =\int_{X} g(x) g(x)^{*} d \mu(x)=\|g\|^{2} .
\end{aligned}
$$

As $\left\{L_{t}: t \in T\right\}$ is abelian and $H_{\lambda}$ is finite dimensional, there exists a basis for $H_{\lambda}$ of eigenfunctions of $\left\{L_{t}: t \in T\right\}$. Hence there exists a spatial eigenfunction $f$ of $\left\{L_{t}: t \in T\right\}$ in $\mathscr{B}(X)$ which is not equal to a constant a.e. $(\mu)$. By Lemma $2.4 f$ is not equal to a constant a.e. Q.E.D.

Note that the proof of Theorem 2.5 shows that without assumption (b) (i.e., $T$ arbitrary), there exist finite-dimensional invariant subspaces of $\mathscr{B}(X)$ containing nonconstant functions.

We next note that if $X$ is compact, the assumption of closed ergodicity in Theorem 2.5 is no more general than the assumption of just ergodicity.

2.6. Proposition. If $X$ is a compact metrizable space and if there exists a closed ergodic invariant probability measure on the Borel sets of $X$ whose support is $X$, then there exists an ergodic probability measure on the Borel sets of $X$ whose support is $X$.

Proof. Let $\mu$ be a closed ergodic probability measure whose support is $X$. Then $\mu(A)=\int_{\mathscr{E}} \nu(A) d \tilde{\mu}(\nu)$ where $\tilde{\mu}$ is a Borel probability measure on the class of ergodic measures $\mathscr{E}$ (cf. [12]). Let $U$ be a nonempty open set and $A=\{\nu \in \mathscr{E}: \nu(U)=0\}$. If $\tilde{\mu}(A)>0$, then using the assumption that $X$ is second countable, $\nu(\mathcal{O}(U))=0$ for all $\nu \in A$ and hence $0<\mu(\mathcal{O}(U))=\int_{A^{\prime}} \nu(\mathcal{O}(U)) d \tilde{\mu}(\nu) \leqq \tilde{\mu}\left(A^{\prime}\right)<1$. Therefore $\mathcal{O}(U)^{\prime}$ is a 
proper closed invariant set with nonzero measure which is a contradiction. Hence $\tilde{\mu}(A)=0$. Since $X$ is second countable, $\{v: \nu(U)>0$ for every nonempty open set 0$\}$ has positive $\tilde{\mu}$ measure and thus is not empty. Q.E.D.

2.7. ExAmple. The following is an example of a transformation group for which (a) and (b) of Theorem 2.5 hold, but for which the converse of Theorem 2.3 fails.

Let $X$ be the space of all equivalence classes (modulo null functions) of measurable functions on $[0,1]$ with Lebesgue measure $\mu$. Let

$$
\rho(f, g)=\int_{0}^{1} \frac{|f-g|}{1+|f-g|} d \mu .
$$

Then $X$ is a separable metric group with no nontrivial continuous characters (cf. [9, pp. 370-372]). Let $T=X$ and $g \pi^{f}=g+f$. Since $\rho\left(f \pi^{h}, g \pi^{h}\right)=\rho(f, g)$, it is easy to see that $(X, T, \pi)$ is not weakly mixing. Since $T$ is metric, every eigenvalue of an eigenfunction is continuous. Using minimality, it follows that $(X, T, \pi)$ has only constant a.e. eigenfunctions.

The next theorem exhibits a class of transformation groups which satisfies (a) and (b) of Theorem 2.5, but not (c), and for which the converse to Theorem 2.3 holds.

2.8. THEOREM. Let $T$ be abelian and $X$ be nontrivial and contain an isolated point. Then $(X, T, \pi)$ is not weakly mixing and there exist nonconstant eigenfunctions in $\mathscr{B}(X)$.

Proof. It follows that $(X, T, \pi)$ is not weakly mixing since $\{(x, y): x=y\}$ is a proper closed invariant set with nonempty interior. Let $x$ be an isolated point and $P_{x}=\left\{t: x \pi^{t}=x\right\}$. Then $P_{x}$ is a subgroup of $T$. If $P_{x}=T, 1_{\{x\}}$ is a nonconstant a.e. invariant function. If $P_{x} \neq T$, let $\chi$ be a nontrivial character of $T / P_{x}$. Define $f$ by

$$
\begin{aligned}
f(y) & =\chi(c(t)) & & \text { if } y=x \pi^{t}, \\
& =0 & & \text { if } y \notin \mathcal{O}(x),
\end{aligned}
$$

where $c$ is the canonical map from $T$ onto $T / P_{x}$. It is easily verified that $f$ is well defined and has the desired properties. Q.E.D.

Finally we give an example where conditions (a) and (c) of Theorem 2.5 hold but the converse of Theorem 2.3 fails.

2.9. ExAmple. Let $X=A_{5}$, the alternating group on five letters, $T=X$, and $f \pi^{g}=g \circ f$. Then $X$ is compact metric and $T$ has no nontrivial characters since it is nonabelian and simple [8, Theorem 5.4.3]. Since $(X, T, \pi)$ is minimal, it follows as in Example 2.7 that $(X, T, \pi)$ is not weakly mixing but has no nonconstant a.e. eigenfunctions.

We now note a case in which the hypotheses of Theorem 2.5 are satisfied. The combination generalizes Furstenberg's result. 
2.10. Theorem. Suppose that $X$ is compact metrizable, $T$ is abelian and $(X, T, \pi)$ is minimal. Then the conditions (a), (b), and (c) of Theorem 2.5 hold.

Proof. It follows by the Markov-Kakutani Theorem [3, p. 456] that $X$ supports an invariant probability measure. By minimality and compactness, every nonempty open set has positive measure, and hence the support is $X$. The conclusion follows by applying Lemma 2.4. Q.E.D.

If $X$ is a separable metrizable Baire space, $T$ is abelian and $(X, T, \pi)$ is uniquely ergodic (i.e., $(X, T, \pi)$ supports a unique finite invariant probability measure on the Borel sets of $X$ whose support is $X$ ), then the conclusion of Theorem 2.10 holds. Note that if $X$ is compact, then such a transformation group is necessarily minimal, and we can apply Theorem 2.10 .

The authors conjecture that the converse of Theorem 2.3 holds when $X$ is compact metrizable and $T$ is abelian.

The following corollary summarizes the results concerning minimal transformation groups.

2.11. Corollary. Suppose that $X$ is compact metrizable, $T$ is abelian and $(X, T, \pi)$ is minimal. Then the following are equivalent:

(a) $(X, T, \pi)$ is weakly mixing.

(b) Every (spatial) eigenfunction $f \in \mathscr{B}(X)$ is a constant a.e.

(c) Every continuous spatial eigenfunction is a constant.

Proof. The implication (a) implies (b) follows from Theorem 2.3. The proof of (b) implies (c) is obvious. To show that (c) implies (a), suppose $(X, T, \pi)$ is not weakly mixing. Then by Theorem 2.5 and 2.10 , there exists a nonconstant spatial eigenfunction $f \in \mathscr{B}(X)$. But a previously mentioned result of Kakutani's (see Lemma 2.4) implies that any point whose orbit is dense in some nonempty open set is a continuity point of any spatial eigenfunction in any Baire space. This yields in our case that $f$ is continuous. Q.E.D.

3. Some properties of weakly mixing minimal transformation groups. In this section, we use Corollary 2.11 to study minimal transformation groups. Unless otherwise noted, $(X, T, \pi)$ will denote a continuous transformation group with $X$ compact metrizable. If $d$ is a compatible metric of $X$, we let $P$ denote the proximal

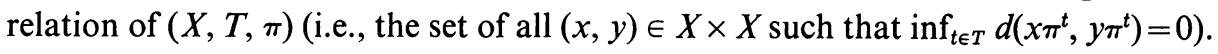
Moreover, $\mathcal{O}(x)$ will denote the orbit of $x \in X$. See [5] for a general reference.

We first examine the algebraic structure of $P$.

3.1. Proposition. Let $X$ be nontrivial and $T$ be abelian. Suppose that $(X, T, \pi)$ is weakly mixing and minimal. Then $P$ is not an equivalence relation.

Proof. Choose $x \in X$ and $t \in T$ such that $x \pi^{t} \neq x$. Suppose $\left(x, x \pi^{t}\right) \in P$. Since $T$ is abelian, it follows that $(X \times X, T, \pi \times \pi)$ is almost periodic at $\left(x, x \pi^{t}\right)$. Then the 
minimal set $\mathcal{O}\left(x, x \pi^{t}\right)^{-}$intersects the diagonal and hence $x=x \pi^{t}$. It follows that $\left(x, x \pi^{t}\right) \notin P$.

Now suppose $P$ is an equivalence relation. Then by [15], $P=L$, the syndetically proximal relation (cf. [2]). Since $(X, T, \pi)$ is weakly mixing, then $\mathcal{O}(y, z)^{-}=X \times X$ for some $(y, z)$ by [7, Theorem 9.20] (the hypothesis that $T$ be generative is not needed). Thus, $P=X \times X$ by [2, Theorem 3], which is a contradiction. Q.E.D.

The topological structure of $P$ is given by the next remark.

3.2. REMARK. Let $(X, T, \pi)$ be weakly mixing. Then $P$ is comeager and so is $x P=\{y:(x, y) \in P\}$ for $x$ in a comeager subset of $X$.

Proof. Since every point in $X \times X$ with dense orbit is in $P$, it follows by [7, Theorem 9.20] that $P$ is comeager. Hence $P^{\prime}$ is meager and so is $x\left(P^{\prime}\right)=\{y:(x, y) \notin P\}$ for $x$ in a comeager set by [11, Corollary 1, p. 222]. The result follows by taking complements. Q.E.D.

An interesting question is to determine what minimal transformation groups have nontrivial structure group (cf. [5, Definition 14]). We now answer this question for abelian $T$.

3.3. LEMMA. Suppose that $(X, T, \pi)$ is ergodic. Then every nonconstant continuous spatial eigenfunction $f \in \mathscr{B}(X)$ induces a transformation group homomorphism onto a minimal continuous transformation group $(Y, T, \rho)$, where $Y$ is nontrivial and the action is equicontinuous.

The straightforward proof of this lemma is omitted. Note that the eigenvalues for such $f$ are always continuous. Moreover, Lemma 3.3 holds when $X$ is simply compact.

3.4. Theorem. Let $T$ be abelian and $(X, T, \pi)$ be minimal. Then $(X, T, \pi)$ is weakly mixing if and only if the structure group $\Gamma$ is trivial.

Proof. Suppose $(X, T, \pi)$ is weakly mixing. Then by Remark 3.2 and [5, Theorem 2], the distal and hence the equicontinuous structure relation are both $X \times X$. Thus, $\Gamma$ is the enveloping semigroup of a one point space and is trivial.

Now suppose $(X, T, \pi)$ is not weakly mixing. Then by Corollary 2.11 and Lemma 3.3, some equicontinuous tranformation group with nontrivial phase space is a homomorphic image of $(X, T, \pi)$. Thus the equicontinuous structure transformation group is nontrivial minimal. It follows that $\Gamma$ is nontrivial. Q.E.D.

As a corollary, we note that if $P$ is an equivalence relation or $P$ is not dense in $X \times X$, then $\Gamma$ is nontrivial by Proposition 3.1 and Remark 3.2 respectively.

Another interesting question is the existence of minimal discrete or continuous flows on spheres. It follows by [6, Theorem 11.1] that no such distal minimal flows exist for arbitrary locally compact abelian $T$ and by [1, Corollary 1] that no such locally almost periodic minimal continuous flows exist. We now show that minimal discrete or continuous flows on spheres must be weakly mixing. Since by Proposition 2.1 $P$ cannot even be an equivalence relation, this generalizes both of the above results. 
3.5. Theorem. Let $X$ be connected and $T$ be abelian. Suppose that $(X, T, \pi)$ is minimal and every continuous map $g: X \rightarrow S^{1}$ (the unit circle) is null-homotopic (or, equivalently, $H^{1}(X)=0$ in Cech cohomology). Then $(X, T, \pi)$ is weakly mixing.

Proof. Suppose $(X, T, \pi)$ is not weakly mixing. Then by Corollary 2.11 , there exists a nonconstant continuous spatial eigenfunction $f \in \mathscr{B}(X)$. We can assume $|f(x)|=1$ for every $x \in X$. It follows from the theory of covering spaces (see [14, p. 103] for example) that $f(x)=e^{i g(x)}$, where $g$ is a continuous real-valued function. The proof is then completed as in [6, Theorem 11.1].

3.6. REMARK. (1) If $X$ is connected, simply-connected, locally path-connected, then $X$ satisfies the hypothesis of Theorem 3.5 (cf. [14, p. 103]).

(2) The theorem is clearly false if $X$ is merely connected. Moreover, in (1) we cannot drop the hypothesis of connectedness. To see this, let $X$ be simply-connected locally path-connected and $(X, \phi)$ be a minimal discrete flow. Letting $Y=X_{1}+X_{2}$, where $X_{i}=X$, and defining $\psi: Y \rightarrow Y$ by $\psi(x, 1)=(x, 2), \psi(x, 2)=(\phi(x), 1)$, then $Y$ is simply-connected locally path-connected and $(Y, \psi)$ is minimal. However, $(Y, \psi)$ is not weakly mixing since $\left(Y, \psi^{2}\right)$ is not ergodic [10, Theorem 3.5]. In particular, one can choose the trivial flow for $(X, \phi)$.

(3) In addition to holding for the simply-connected spheres and complex or quaternionic projective planes, Theorem 3.5 holds for real projective planes $\mathscr{P}^{n}$, $n \geqq 2$, and lens spaces.

(4) Theorem 3.5 is false if $T$ is nonabelian. To see this, let $X=S^{2}$, the 2-sphere, and $T=S O(3)$, the rotation group on $X$, with its discrete topology. Choosing the natural action $\pi$, it is clear that $(X, T, \pi)$ is equicontinuous minimal and hence not weakly mixing.

One can prove Theorem 3.5 with no assumptions on the joint continuity of $(x, t)$ $\rightarrow x \pi^{t}$ since Corollary 2.11 holds in this case. Also, since eigenvalues of spatial eigenfunctions are continuous if $t \rightarrow x \pi^{t}$ is continuous, one can then show Lemma 3.3 under this hypothesis. However, if $T$ is locally compact, it follows by [4, Theorem 1] that the continuity of $t \rightarrow x \pi^{t}$ is equivalent to $(X, T, \pi)$ being a continuous transformation group.

4. Eigenvalue theorems for minimal transformation groups. In this section, we show how replacing a.e. equality by spatial equality in $\mathscr{B}(X)$ yields analogous eigenvalue theorems for minimal transformation groups. Again, $X$ will simply denote a Baire space and $(X, T, \pi)$ a transformation group.

4.1. THEOREM. The transformation group $(X, T, \pi)$ is minimal if and only if every spatially invariant function in $B(X)$ is a constant.

Proof. The "if" part is clear. For the converse, suppose that $(X, T, \pi)$ is minimal and $f \in \mathscr{B}(X)$ is a nonconstant spatially invariant function. Choose $x, y \in X$ such that $f(x) \neq f(y)$. Since $\mathcal{O}(x), \mathcal{O}(y)$ are dense and $f$ is constant on orbits, then $f(x)$ $=f(z)=f(y)$ for some $z \in C(f)$. This is a contradiction. Q.E.D. 
4.2. Corollary. If $(X, T, \pi)$ is weakly mixing and minimal, then every spatial eigenfunction in $\mathscr{B}(X)$ is a constant.

Proof. Use Theorem 2.3, noting that every spatial eigenfunction is continuous (see Corollary 2.11).

If $X$ is compact metrizable and $T$ abelian, then the converse of Corollary 4.2 follows from Theorem 4.1 and Corollary 2.11.

We now consider discrete flows. The following result is analogous to [10, Theorem (3.5)].

4.3. TheOREM. Consider the discrete flow $(X, \phi)$ and let $m \neq 0$. Then $\left(X, \phi^{m}\right)$ is minimal if and only if for all $f \in \mathscr{B}(X), f \phi=\lambda f$ and $\lambda^{m}=1$ implies $f$ is a constant.

Proof. The "only if" part follows from Theorem 4.1. For the converse, assume $m>0$ and $\left(X, \phi^{m}\right)$ is not minimal. Let $C$ be a proper closed $\phi^{m}$-invariant subset. If $C^{0} \neq \varnothing$, we can apply [10, Theorem (3.5)]. If $C^{0}=\varnothing$, we recursively define a sequence $\left(D_{k} \mid k \geqq 0\right)$ by

$$
\begin{aligned}
D_{0} & =C, \\
D_{k+1} & =D_{k}-\phi^{k+1} D_{k}, \quad \text { if } D_{k}-\phi^{k+1} D_{k} \neq \varnothing, \\
& =\varnothing, \quad \text { if } D_{k}-\phi^{k+1} D_{k}=\varnothing, \text { and stop the sequence. }
\end{aligned}
$$

Then we can inductively show that $\phi^{m}\left(D_{k}\right)=D_{k}$ and $D_{k}, \phi D_{k}, \ldots, \phi^{k} D_{k}$ are disjoint. Moreover, $D_{m}$ is never defined. The proof is completed in the same way as in [10, Theorem (3.5)]. Q.E.D.

As a corollary, we note that $(X, \phi)$ is totally minimal iff the only root of unity which is an eigenvalue of $\mathscr{B}(X)$ is 1 , and its eigenfunctions are constants.

4.4. REMARK. Suppose $(X, \phi)$ is minimal and $m \neq 0$. Then $\left(X, \phi^{m}\right)$ is minimal iff $\left(X, \phi^{m}\right)$ is ergodic.

Proof. Suppose $m>1$ and $\left(X, \phi^{m}\right)$ is not minimal. Let $C$ be a proper closed $\phi^{m}$-invariant subset. If $D=\bigcup_{i=0}^{m-1} \phi^{i} C, D$ is closed $\phi$-invariant and hence $X=D$ by minimality. Thus, $\left(\phi^{i} C\right)^{0} \neq \varnothing$ for some $i$. It follows that $C^{0} \neq \varnothing$ and $\left(X, \phi^{m}\right)$ is not ergodic. The other way is trivial. Q.E.D.

\section{REFERENCES}

1. H. Chu and M. Geraghty, The fundamental group and the first cohomology group of a minimal set, Bull. Amer. Math. Soc. 69 (1963), 377-381.

2. J. P. Clay, Proximity relations in transformation groups, Trans. Amer. Math. Soc. 108 (1963), 88-96.

3. N. Dunford and J. Schwartz, Linear operators, Vol. I, Interscience, New York, 1958.

4. R. Ellis, Locally compact transformation groups, Duke Math. J. 24 (1957), 119-126.

5. R. Ellis and W. Gottschalk, Homomorphisms of transformation groups, Trans. Amer. Math. Soc. 94 (1960), 258-271.

6. H. Furstenberg, The structure of distal flows, Amer. J. Math. 85 (1963), 477-515.

7. W. Gottschalk and G. Hedlund, Topological dynamics, Amer. Math. Soc. Colloq. Publ., Vol. 36, Amer. Math. Soc., Providence, R. I., 1955. 
8. M. Hall, The theory of groups, Macmillan, New York, 1959.

9. E. Hewitt and K. A. Ross, Abstract harmonic analysis, Vol. I, Academic Press, New York, 1963.

10. H. Keynes and J. Robertson, On ergodicity and mixing in topological transformation groups, Duke Math. J. (to appear).

11. C. Kuratowski. Topologie, Vol. I, Monogr. Mat., Warsaw, 1952.

12. R. R. Phelps, Lectures on Choquet's theorem, Van Nostrand, Princeton, N. J., 1965.

13. F. Riesz and B. Sz.-Nagy, Functional analysis, Ungar, New York, 1955.

14. E. Spanier, Algebraic topology, McGraw-Hill, New York, 1966.

15. T. S. Wu, Proximal relations in topological dynamics, Proc. Amer. Math. Soc. 16 (1965), 513-514.

University of CALIFornia,

Santa Barbara, California 